\title{
Endoscopic removal of localized gastric amyloidosis
}

Amyloidosis is characterized by the extracellular accumulation of fibrillar proteins, which commonly shows systemic involvement. Localized amyloidosis is rare, especially regarding the stomach. The lesion may cause paresis, constipation, and bleeding [1-3]. We saw a case of gastric localized amyloidosis with bleeding, in which endoscopic resection was successfully done as a treatment for anemia.

A 77-year-old woman with chronic liver disease was referred to our hospital with anemia. Esophagogastroduodenoscopy revealed a flat, depressive lesion at the greater curvature of the lower gastric body ( $\bullet$ Fig. 1). The lesion bled easily after air inflation ( $\bullet$ Fig. 2).

Because the anemia could not be improved with conservative treatment, the lesion was removed by endoscopic submucosal dissection ( $\bullet$ Fig.3). The lesion was $46 \times 28 \mathrm{~mm}$, and pathological examination showed amyloid deposits in the mucosal and submucosal layer ( Fig.4). The material stained positive with direct fast scarlet (DFS). The positivity with DFS staining was not affected by pretreatment with potassium permanganate, suggesting the possibility of amyloid light-chain (AL) type amyloid protein. Serum amyloid A was normal $(5.5 \mu \mathrm{g} / \mathrm{mL}$; normal range $0-8.0 \mu \mathrm{g} / \mathrm{mL}$ ).

After treatment, the ulcer had formed a scar, and the patient's anemia improved (๑ Fig.5).

Endoscopy_UCTN_Code_CCL_1AB_2AD_3AF

Competing interests: None

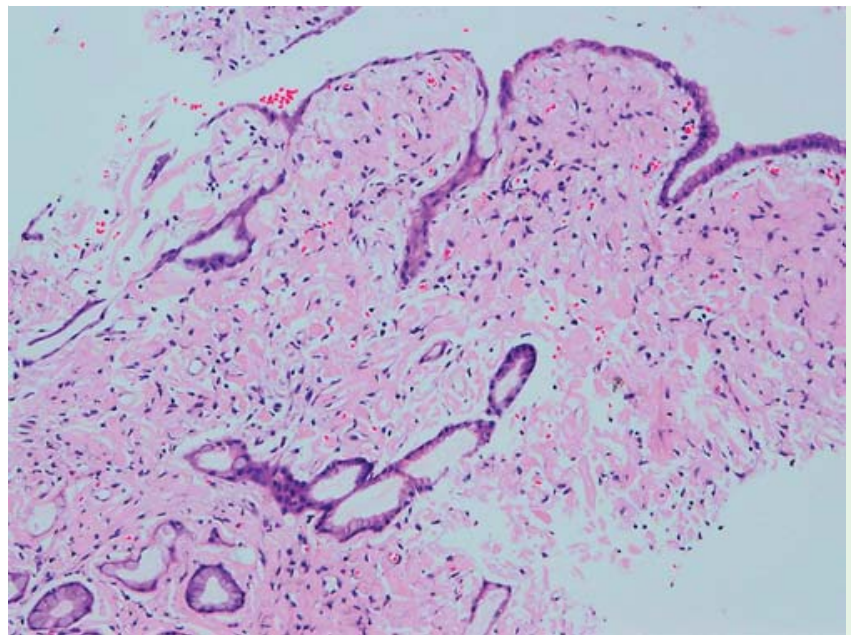

Fig.4 Pathological examination revealed massive amyloid deposits in the mucosal and submucosal layer. (Hematoxylin and eosin, $\times 400$.)

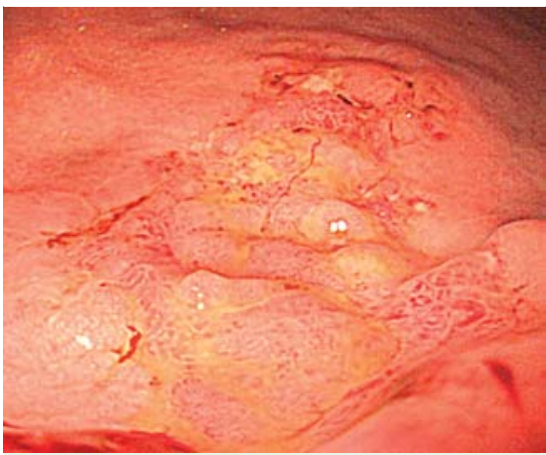

Fig. 1 Endoscopy showed a flat, slightly depressed lesion at the gastric body.

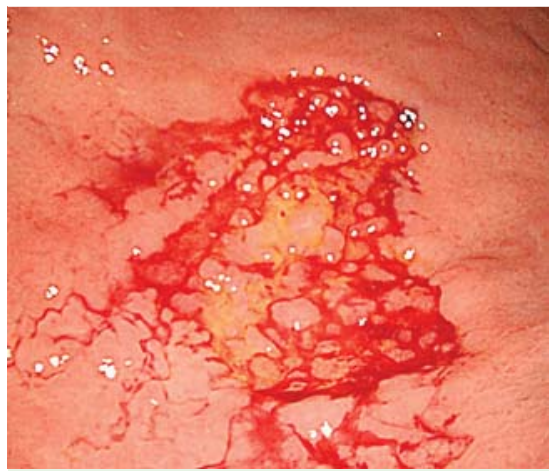

Fig. 2 Bleeding occurred easily after air inflation.

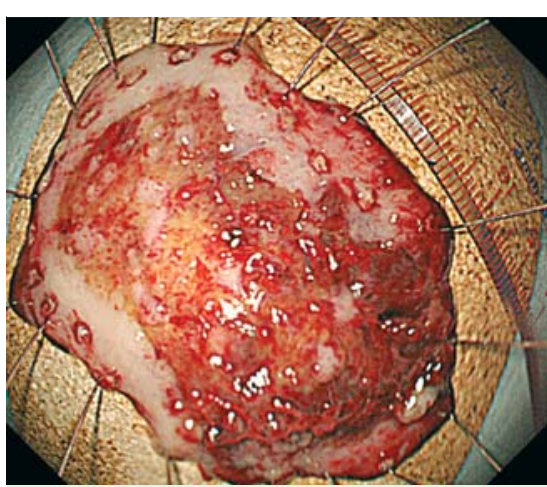

Fig. 3 En bloc removal of the lesion by endoscopic submucosal dissection was successfully carried out.

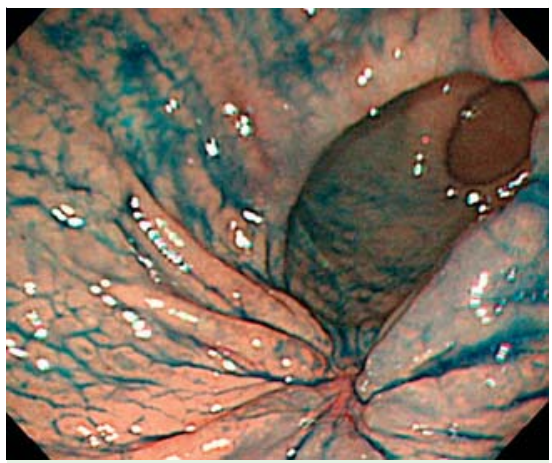

Fig. 5 Gastric ulcer 3 months after local amyloidosis had been removed. 